International Journal of Forensic Science \& Pathology (JJFP)

ISSN 2332-287X

\title{
Partial Autopsy - A Second Thought
}

Chattopadhyay S

Editorial

Associate Professor \& Head, Department of Forensic Medicine, Murshidabad Medical College, Berhampore, West Bengal, India

\section{*Corresponding Author:}

Dr. Saurabh Chattopadhyay,

Associate Professor \& Head, Department of Forensic Medicine, Murshidabad Medical College, Berhampore, West Bengal, India.

E-mail: chattopadhyaydrs@rediffmail.com

Received: January 25, 2014

Published: February 20, 2014

Citation: Chattopadhyay S (2014) Partial Autopsy - A Second Thought. Int J Forensic Sci Pathol. 2(1e), 1. doi: http://dx.doi. org/10.19070/2332-287X-140002e

Copyright: Chattopadhyay $\mathbf{S}^{\circ} 2014$. This is an open-access article distributed under the terms of the Creative Commons Attribution License, which permits unrestricted use, distribution and reproduction in any medium, provided the original author and source are credited.

One of the objectives of medicolegal autopsy is to find out the cause of death. To fulfill such an objective it is worthy that a detailed and meticulous autopsy procedure is conducted. In conventional practice, each and every body cavity is dissected to find out the presence of any abnormality. Such an extensive autopsy procedure is not without its drawbacks. The autopsy surgeon's focus should not be restricted only to find out the cause of death. He must also be aware of the sentiments of the bereaved family members and relatives of the deceased. In some of the centers in developing countries the case load is about 15-20 cases per day. Extensive dissection of the body not only causes disfiguration to some extent but also requires a lot of time for proper reconstruction and suturing so that the body is in a sober state before handing it over to the family members. Time constraint is an important factor for the centers with a large case load. The mental agony and pain of the family members waiting to complete the last rights and rituals is beyond any words of explanation.

Medical science is a science based not only on knowledge, evidence and justification but also takes into consideration human values and ethics. It is beyond any doubt that dissection of every body cavity is not fruitful in all cases. Consider a case of road traffic accident with crush injury on the head due to run over, without any significant external injury on the chest or abdomen. In such a case dissection of the abdomen to find out an incidental hypernephroma or cirrhosis of the liver does not add any worth of information to come to a conclusion regarding the cause of death. Similarly in cases of hanging, stab injury of the abdomen or bullet injury over the head a focused dissection of the affected body region - partial autopsy, may be worth considering. An opinion regarding the cause of death from partial autopsy may be a point of argument for the lawyers in the court of law but it seems insignificant when considering the ethical values of medical science.

In un natural deaths where the cause of death is quite obvious the concept of partial autopsy may be accepted. It is the knowledge and discretion of the autopsy surgeon that is to be relied upon. The autopsy surgeon should decide whether a complete or partial autopsy is necessary in the particular case. Only those body cavities should be dissected where the autopsy surgeon expects some findings to explain the cause of death. In the process if it is necessary or if the situation demands he may proceed for a complete autopsy. A focused approach not only reduces the time for autopsy but also helps to examine in detail the body region with expected findings.

In pathological autopsy the concept of partial autopsy has been well accepted $[1,2]$. Based on the history of the case and medical records the pathologist decides on the system to be investigated by autopsy. Very similarly in case of medicolegal autopsy also, history from the family members, police inquest and medical records can be a basis on which a partial autopsy may be conducted. In recent years minimally invasive autopsies have been advocated in high risk cases with HIV / Hepatitis C infections [3].

Thus a reduction in the number of complete postmortem examinations may not be impossible. However it must not be a routine practice as there are limitations in the proposed method. It must always be kept in mind that lapses in medicolegal autopsy may have far reaching consequences in terms of legal issues. On the other hand legal issues cannot be a bar to provide service to mankind with a humane touch. A balance should be made judging the merits and demerits of the case and partial autopsy may be undertaken in good faith.

\section{References}

[1]. Suchil BL, Osornio VAR. Cardiovascular pathology and the partial autopsy. The experience at the Instituto Nacional de Cardiología Ignacio Chávez of Mexico. Rev Esp Cardiol. 1991; 44(10): 634-8.

[2]. Erica C. Savage, Robert A. Robinson, and Patricia Kirby. Utility of a Partial Autopsy: A Case Report of Congenital Laryngeal Atresia. Pediatric and Developmental Pathology: 2013; 16(3): 229-231.

[3]. Fryer EP, Traill ZC, Benamore RE, Roberts ISD. High risk medicolegal autopsies: is a full postmortem examination necessary? Clin Pathol. 2013; 66:1-7. 